\title{
Avaliação das características do TemaTres e Multites para o controle de autoridades nas bibliotecas universitárias
}

Evaluación de las características de TemaTres y Multites para el control de autoridades en bibliotecas universitarias

An assessment of TemaTres and Multites characteristics for authority control in academic libraries

\section{Mariângela Spotti Lopes FUJITA, Luciana Beatriz Piovezan dos SANTOS, Maria Carolina Andrade CRUz, Walter MOREIRA}

UNESP, Campus de Marília, Av. Hygino Muzzi Filho, 737, Marília, SP, 17525-300, fujita@marilia.unesp.br, nanipiovezan@gmail.com, maria.andradeecruz@gmail.com, Walter.moreira@marilia.unesp.br

\begin{abstract}
Resumen
Se analizaron dos softwares para la construcción y mantenimiento de tesauros, TemaTres y MultiTes, de cara a facilitar el control de autoridades en bibliotecas universitarias. Los resultados apuntan que ambos softwares cumplen con la mayoría de los requisitos, y que TemaTres se emplea ya en sistemas de bibliotecas universitarias en la recuperación por materia de registros bibliográficos del catálogo en línea. Sin embargo, se recomienda la aplicación experimental de los dos softwares para el análisis de su comportamiento operativo tanto en la construcción del tesauro como en su uso con registros de autoridad y en la recuperación por materias.
\end{abstract}

Palabras clave: Tesauros. Software de gestión de tesauros. Catálogos en línea. Control de autoridades. Bibliotecas.

\section{Introdução}

Diante ao grande fluxo de produções tecnológicas e científicas, os centros de informação, como as bibliotecas, buscam aprimoramento no armazenamento e recuperação da informação. O método para contribuir com essa situação resultou na criação de recursos próprios para o controle de vocabulário no tratamento da informação: as linguagens de indexação.

De acordo com Baritè (2011, p. 127, tradução nossa) o controle de vocabulário "contribui com a seleção, depuração, formalização e normalização da terminologia, incluindo, o estabelecimento recíproco entre os termos, considerando critérios semânticos, linguísticos e disciplinares". Com essas características, compreende-se que o controle de vocabulário em um sistema de informação, favorece tanto o sistema de organização da informação quanto o sistema de recuperação.

A linguagem de indexação é uma ferramenta de controle de vocabulário utilizada pelo indexador na etapa de tradução dos conceitos do processo

\begin{abstract}
Two thesaurus management software applications, TemaTres and MultiTes, were assessed regarding their suitability for controlling authorities in university libraries. The results indicate that both software applications comply with most of the requirements, and that TemaTres is already used in university library systems for retrieving bibliographic records from online catalogues. However, an experimental assessment of their operational behavior both in the construction of thesauri, in its use with authority records and in subject retrieval is recommended.
\end{abstract}

Keywords: Thesauri. Thesauri management software. Authority control. Online catalogues. Libraries.

da indexação e na estratégia de busca pelo usuário. Compreende a mediação entre a linguagem natural extraída dos documentos e a do usuário na estratégia de busca, buscando assim contribuir para a busca e recuperação do documento (Martins, 2013).

De acordo com a norma norte americana ANSI/NISO Z39.19 as linguagens de indexação configuram-se em (American National Standards Institute/ National Information Standards Organization 2005, p. 11, tradução nossa)

[...] vocabulário controlado ou um sistema de classificação com regras para sua aplicação. Uma linguagem de indexação é utilizada para a representação dos conceitos tratados nos documentos [objeto de conteúdo] e para a recuperação de tais documentos [objetos de conteúdo] de um armazenamento de informações e de sistema de recuperação

Ainda de acordo com a norma norte americana, as linguagens de indexação configuram-se nos seguintes formatos (seguindo a lógica de complexidade): as listas, os anéis de sinônimos, as 
taxonomias e os tesauros. Neste artigo, pretende-se abordar softwares para a gestão especificamente dos tesauros.

Compreende-se a importância dos tesauros nas palavras de Cervantes (2004, p. 35) ao dizer que o tesauro é considerado como o mais adequado para o tratamento e recuperação da informação, pois "além de estabelecer relações entre os termos utilizados, denominados de preferidos e não preferidos, este instrumento permite uma visão sistêmica da área - objeto de representação". Os avanços tecnológicos vêm aprimorando de modo significativo as ferramentas destinadas à representação e recuperação da informação. Em relação aos sistemas de informação, percebe-se a necessidade de investir em novas tecnologias para garantia do bom desempenho de seus sistemas de recuperação.

Os tesauros, assim como outras linguagens mais complexas, necessitam dos softwares para a sua construção e manutenção. Em vista das necessidades de recuperação por assunto em catálogos online de bibliotecas, cujos padrões descritivos e temáticos utilizam metadados para a construção de registros bibliográficos e registros de autoridade, torna-se fundamental a construção e manutenção de linguagens de indexação com uso de softwares acessíveis e que suportem grande quantidade de termos e seus relacionamentos, tendo em vista que as bibliotecas, em sua maioria, possuem coleções bibliográficas em todas as áreas de conhecimento. Cabe destacar também, como requisito mínimo desejável ao software, as condições que este oferece para a construção e manutenção de tesauros que abriguem aspectos de interoperabilidade em ambientes multilíngues.

Para a identificação e acesso à softwares de gestão de tesauros, destacam-se os trabalhos de reunir em uma lista os recursos existentes com descrições técnicas e operacionais, tais como o de Leonard D. Will no site "TaxoBank: Access, deposit, save, share, and discuss taxonomy resources" (1) e o da Biblioteca da Universität Basel associada à International Society of Knowledge Organization (ISKO) "Blasel Register of Thesauri, Ontologies \& Classifications" (2) que disponibilizam informações sobre os softwares de gestão de tesauros.

Consideramos de fundamental importância para o desenvolvimento de avaliação de softwares de gestão de tesauros os resultados dos trabalhos de Campos et al (2006), de Santos et al. (2013) e Martínez González e Alvite Díez (2014). O primeiro trabalho "Estudo comparativo de softwares de construção de tesauros" realizou comparação com seis softwares de construção de tesauros, entre eles o TemaTres e o MultiTes. O segundo trabalho "Elaboração de vocabulário controlado em formato SKOS usando Tematres: implicações metodológicas e web-semântica" incluiu experiência de construção de um tesauro sobre "cerveja" utilizando os softwares TemaTres e o MultiTes. O terceiro trabalho apresenta uma análise sistemática de seis softwares para a gestão de tesauros, entre eles o TemaTres e o MultiTes, mediante aplicação de um conjunto de critérios estabelecidos a partir de sistematização de literatura que considera o propósito e funcionalidade dos aplicativos, integração com outros sistemas de informação ou interoperabilidade da informação e a capacidade de combinar diferentes fontes de tesauros. A proposta é analisar os dois softwares para construção e manutenção de tesauros citados nos trabalhos de Campos et al (2006), de Santos et al. (2013) e Martínez González e Alvite Díez (2014) o TemaTres e o MultiTes.

\section{O controle de registros de autoridades em catálogo online de biblioteca: funções e finalidades}

Partindo da idéia de Jewett (1853) de catalogação cooperativa e uso de recursos tecnológicos, a Library of Congress (LC) criou a maior Central de Catalogação cooperativa. A introdução da tecnologia computacional na década de 1960 e o desenvolvimento do formato MARC para padronização de registros bibliográficos e formatos de intercâmbio reflete-se até os dias atuais nas bibliotecas e centros de informação.

O desenvolvimento de tecnologias de software e hardware juntamente com a Internet proporcionou às centrais de catalogação cooperativa disponibilizarem seus catálogos online, acelerando a troca de informações. Para propiciar o intercâmbio de informações, foram criados, junto ao formato MARC, os protocolos Z39.50 (3) e ISO2709 (4) que orientam sobre a estrutura de um registro e a transferência de dados bibliográficos.

Quando um registro bibliográfico é criado, o catalogador faz a descrição bibliográfica do item documentário nos campos devidos e quando se depara com os campos de assunto, recorre à Base de Autoridades Assuntos que mantém os denominados Registros de autoridade que formam, atualmente, o controle de vocabulário em formato MARC21.

Os registros de autoridade destinam-se a conter informação autorizada para padronizar nomes e assuntos a serem usados como pontos de acesso em registros bibliográficos, bem como permitir os relacionamentos entre eles. Compõem uma base de dados que é consultada, tanto pelo catalogador ao atribuir os termos que representam assuntos, quanto automaticamente pelo próprio sistema para correção de termos e

Fujita, M. S. L.; Santos, L. B. P. Dos; Cruz, M. C. A.; Moreira, W. Avaliação das características do TemaTres e Multites para o controle de autoridades nas bibliotecas universitárias. // Scire. 23:2 (jul.-dic. 2017) 63-73. ISSN 1135-3716. 
inclusão de remissivas dentro dos registros bibliográficos. Isso significa que os registros de autoridade têm o objetivo de assegurar a constante padronização da linguagem de indexação de forma automática e podem ser transferidos de uma base de dados para outra na formação dos catálogos de bibliotecas e rede de bibliotecas.

Em seguida, apresenta-se exemplo de registro de autoridade de assunto de termo tópico da ferramenta Classification Web da Library of Congress Subject Headings (LCSH) em formato de ficha terminológica simples e registro MARC.

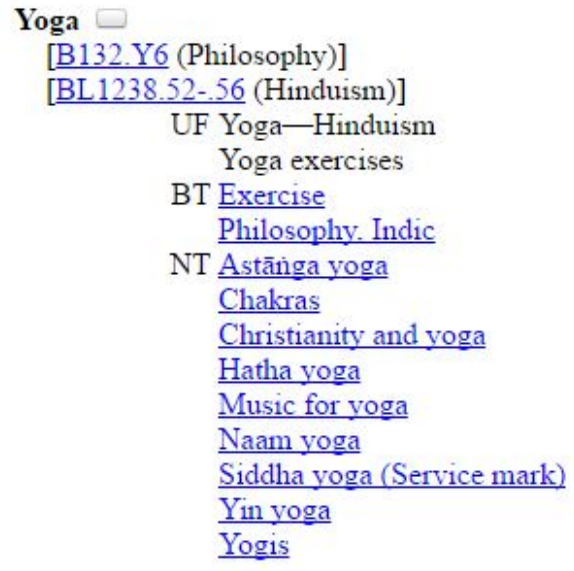

Figura 1. Registro de autoridade em ficha completa (Fonte: Classification Web-LCSH)

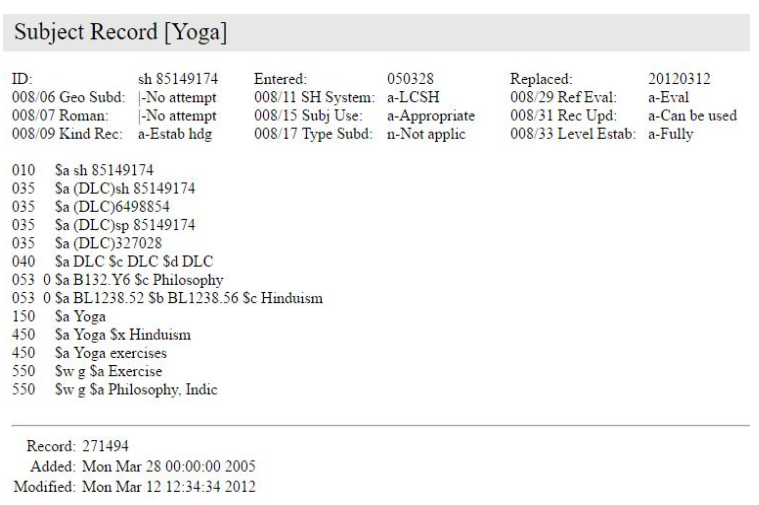

Figura 2 Registro de autoridade em formato MARC21 (Fonte: Classification Web - LCSH)

Ressalte-se que a Library of Congress Subject Headings - LCSH, evoluiu em suas últimas edições com a introdução e uso da nomenclatura de relações semânticas adotadas por tesauro, incluindo uma estrutura lógica de relações hierárquicas anteriormente atrofiada na linguagem. Essa evolução da LCSH, os formatos de intercâmbio de registros bibliográficos e os de autoridades e os catálogos online demonstram que são importantes para a indexação e recuperação por assuntos em catálogos online de bibliotecas.

O controle de registros de autoridades tem sua importância porque estão ligados à própria necessidade de construção de vocabulários controlados legíveis por computador que contribuam para o desenvolvimento da web semântica, sobretudo porque torna possível sua reutilização por outros sistemas de informação (Martins, 2013).

A esse respeito é preciso refletir sobre a importação de registros de autoridade com o necessário controle de vocabulário aliado à construção de linguagens de indexação. Nesse sentido, o controle de autoridades tem duas funções importantes a de manter a coerência da forma usada na representação dos pontos de acesso e das relações entre conceitos. Os registros de autoridade têm esse nome porque foram criados para o controle dos cabeçalhos de autores, mas como os cabeçalhos de assuntos necessitam também manter o controle da forma de termos e de seus relacionamentos com outros termos e conceitos, o registro de autoridade passou a ter função imprescindível na construção de linguagens de indexação.

A importação de registros de autoridade de assunto por catalogadores sem a definição de diretrizes de construção e manutenção de linguagens de indexação contraria a dupla função do controle de autoridades e não propicia a adequação do uso de linguagens de indexação para o controle de vocabulário durante a indexação e durante a estratégia de busca para a recuperação por assuntos em catálogos online. Portanto, é necessário que o software de gestão da construção e manutenção de tesauros a ser utilizado na indexação e recuperação por assuntos em catálogos online tenha condições de fazer a importação dos registros de autoridade para que se mantenha a integridade da linguagem de indexação.

\section{A construção e manutenção de tesauros na perspectiva da normalização para análise de softwares}

No âmbito da Ciência da Informação, a palavra tesauro nomeia o instrumento que reúne um conjunto controlado de termos de um determinado domínio, demonstrando as relações conceituais e terminológicas ou relações semânticas e de equivalência entre estes termos. Tal instrumento tem a função de auxiliar nos processos de representação e de recuperação da informação documentária em domínios específicos, respeitando sua terminologia própria. 
Em obra na qual apresenta aspectos essenciais da construção de tesauros, Broughton (2006, p.4, tradução nossa), define tesauro como:

[...] uma ferramenta usada para a indexação do assunto de documentos. Consiste em uma lista estruturada de termos (geralmente em um campo de assunto específico) que um indexador ou gerenciador de registros pode usar para descrever documentos de modo que os usuários finais possam recuperar itens relevantes ao pesquisar material sobre um determinado assunto.

Na definição apresentada na norma ISO 259641 (2011) enfatiza-se o aspecto estrutural do tesauro. Nesta norma, define-se tesauro como:

Vocabulário controlado e estruturado em que os conceitos são representados por termos, organizados de modo que as relações entre conceitos sejam explicitadas e os termos preferidos sejam acompanhados por termos de entrada para sinônimos ou quasi-sinônimos.

Em nota explicativa para a definição apresentada a referida norma caracteriza o aspecto funcional do tesauro. Considerando-se este aspecto (International Organization for Standardization 259641, 2011, tradução nossa),

Nota: O propósito de um tesauro é orientar tanto o indexador quanto o pesquisador para a seleção do mesmo termo preferido ou de uma combinação de termos preferidos para representar um determinado assunto. Por esta razão, um tesauro é otimizado para a navegabilidade humana e a cobertura terminológica de um dominio.

Em contexto histórico, o aparecimento dos tesauros é relativamente recente e foi determinado pela conjuntura de 1950 e 1960 que apresentou progressos no campo tecnológico e descobertas científicas em diversas áreas do conhecimento, gerando o aumento da produção científica. Com isso, a atenção para os sistemas de organização e recuperação da informação se intensificaram, pois, compreendia a necessidade de buscar ferramentas para o processamento dos documentos, como as linguagens próprias. Nesse período, teve o início do desenvolvimento dos primeiros tesauros (Cintra et al, 2002).

O aparecimento dos primeiros tesauros, da Dupont em 1959, do Defense Technical Information Center (DTIC) (anteriormente Armed Services Technical Information Agency - ASTIA) em 1960, Chemical Engineering Thesaurus em 1961, Thesaurus of Engineering Terms, publicado pelo Engineering Joint Council (EJC) em 1964, influenciou a elaboração das primeiras diretrizes sobre a construção de tesauros, publicadas pelo Commitee on Scientific and Technical Information (COSATI) em 1967, utilizadas para a construção do Thesaurus of Engineering and Scientific Terms (TEST) aem 1967 que serviram de base para a elaboração das primeiras normas sobre construção de tesauros: Guidelines for the Establishment and Development of Monolingual Scientific and Technical Thesauri da UNESCO em 1970 e a ANSI Z39.19 de 1974 da American National Standards Institute (Cervantes e Fujita, 2012; Mendes, Reis e Maculan, 2015).

Em um estudo sobre as origens do processo de construção de tesauros, Cervantes e Fujita (2012) e Campos et al. (2006), citam a figura de Lancaster (1995, p.50) que demonstra graficamente a evolução dos tesauros, considerada por Campos et al. (2006) com duas vertentes, a alfabética, representativa da linha americana, e a sistemática, da linha europeia. Cervantes e Fujita (2012, p. 124), em complementação, consideram que essas "origens podem ser ou parcialmente teóricas, iniciadas pela linha teórica ranghanatiana (classificações bibliográficas), ou parcialmente empíricas, com embasamento aplicado às práticas da indexação alfabética de assunto iniciadas pelas regras de Cutter".

$\mathrm{Na}$ evolução atual das normas estão presentes a Norma Z39.19 - Guidelines for the construction, format, and management of monolingual controlled vocabularies (American National Standards Institute/ National Information Standards Organization, 2005) - proposta desenvolvida pela organização norte-americana National Information Standards Organization (NISO) e a Norma ISO 25964 (2011) "Information and documentation: part 1: thesauri and interoperability with other vocabularies" e mais recentemente a Norma ISO 25964 (2013) "Information and documentation - thesauri and interoperability with other vocabularies - part 2: Interoperability with other vocabularies".

Clarke e Zeng (2012, p.25) esclarecem que a Parte 1 da Norma ISO 25964 "atualiza, revisa e substitui a ISO 2788 e a ISO 5964, bem como algumas partes da BS 8723". Isso significa que, mais uma vez, a atualização abrange várias normas e segue influências de vertentes metodológicas importantes. As normas atuais, como se constata, evoluíram consideravelmente a partir da última edição da ISO 2788:1986 - "Guidelines for the establishment and development of monolingual thesauri" (1986), demonstrada ao final da figura de Lancaster, cujas influências são provenientes tanto da indexação alfabética de assuntos quanto da classificação bibliográfica advindas das duas vertentes metodológicas. Anterior a esta última edição é a norma ISO 5964:1985 "Guidelines for the establishment and development of multilingual thesauri" cuja principal contribuição foi o tratamento de várias línguas em um mesmo recurso. 
Conforme Martins (2013) a norma ANSI/NISO Z39.19 (2005) é a quarta edição da norma ANSI Z39.19-1974 cujo fator determinante de mudança foi a aplicação de tecnologias de informação para desenvolvimento de tesauros eletrônicos em função dos avanços da informação eletrônica e da internet.

O lançamento da norma britânica BS 8723 nos anos de 2005-2008 adianta-se em relação a esses avanços da norma ANSI Z39.19 (2005) e traz diretrizes acerca da interoperabilidade linguística, análise de facetas e interoperabilidade entre linguagens de indexação, incorporadas, na evolução da Norma ISO 25964-1 (2011). Dessa forma, a norma ISO 25964 incorpora em suas diretrizes vários aspectos evolutivos das normas que a precedem: os aspectos de mono e multilinguismo, interoperabilidade linguística, interoperabilidade entre vocabulários controlados, aplicação da análise de facetas na construção do tesauro, definição de protocolos e formatos necessários para o intercâmbio de dados e a proposta de modelização de dados.

De acordo com divulgação das normas na página da NISO (5), a parte 1 da ISO 25964 de 2011 "fornece recomendações para o desenvolvimento e manutenção de tesauros destinados a aplicações de recuperação de informação, seja monolíngue ou multilíngue" com destaque para seu item 14 que é dedicado especialmente a orientações quanto aos requisitos de software para a gestão de tesauros. A parte 2 da ISO 25964-2: 2013 "dá recomendações para o estabelecimento e manutenção de mapeamentos entre vários tesauros, ou entre tesauros e outros tipos de vocabulários". Nesta segunda parte da norma, há interfaces relativas à interoperabilidade com os esquemas de classificação, as taxonomias, os cabeçalhos de assunto, as ontologias, as terminologias, as listas de autoridade de nomes e os anéis de sinônimos.

O avanço existente na norma ISO 25964, em relação às demais normas anteriores, refere-se fundamentalmente à diferenciação entre conceito e termo explicitados por modelos de dados para que as máquinas possam manipular e interpretar dados e realizar processo de inferência (Alvite Diez e Martínez Gonzalez, 2015, Santos, et al., 2013). Isso significa que os conceitos estão representados por termos e que é preciso identificar e descrever tanto as relações que ocorrem entre os conceitos (relações conceituais) quanto as que ocorrem entre os termos (relações terminológicas). Tal proposta nos remete à ideia do tesauro conceitual de Dahlberg (1978) como foi explicado por Campos et al. (2006, p.71):

Este é um tesauro com base em conceitos, onde na sua construção são necessários princípios para o estabelecimento do termo/conceito e das relações entre eles. Neste tipo de tesauro o sentido linguístico não é mais determinante na criação e organização dos termos e sim o seu conteúdo conceitual.

$\mathrm{Na}$ avaliação das normas atuais, além das concepções conceituais que as fundamentam, observa-se que dedicam itens específicos para a gestão e manutenção de tesauros com uso de softwares. A evolução das tecnologias de informação na sociedade humana é notoriamente um fator de mudança e nas bibliotecas não foi diferente mesmo porque, o fluxo de produção científica tem se intensificado cada vez mais e a busca por tecnologias que contribuam para a recuperação da informação criada eletronicamente é imprescindível.

Tal necessidade é constatada por Boccato (2011, p. 5) em sua afirmação de que

Com o crescimento da indústria da informação e, consequentemente, com o advento das bases de dados bibliográficas, houve a necessidade de diretrizes normativas na construção de tesauros disponíveis não só no formato impresso, como também no formato eletrônico, via web.

Os softwares para a construção dos tesauros são, de fato, uma necessidade para elaboração da ferramenta em ambiente digital. Os softwares são necessários pelas "[...] facilidades de armazenamento, manipulação e apresentação dos termos definidos e suas relações" (Campos et al 2006, p. 69).

Na década de 1990, Milstead (1991) apontou a carência de softwares voltados especificamente para contemplar os requisitos básicos para garantir o bom desempenho no sistema de recuperação na base de dados de um tesauro. Hoje, o cenário se modifica e apresenta softwares capacitados para atender as demandas dos sistemas de informação que investem na esfera da recuperação da informação.

O precursor na criação de tesauros assistidos por software no Brasil foi o IBICT. Conforme histórico descrito na página do IBICT (6) software TECER foi desenvolvido no final dos anos 1980 e amplamente distribuído em âmbito nacional. Pode se perceber a necessidade da ferramenta diante do interesse e uso pela comunidade. Apesar disso, por um período de tempo, o software saiu do mercado por falta de atualização. Atualmente, o IBICT decidiu atualizar o software e disponibilizálo para download. Em análise da nova versão do TECER, Mendes, Reis e Maculan (2015, p.62) observam que, "apesar de os estudos anteriores (conforme demonstrado no Quadro 2) já terem identificado diversificada gama de tipos de relacionamentos, podemos notar que o software não foi capaz de atender a essa demanda." O Quadro

Fujita, M. S. L.; Santos, L. B. P. Dos; Cruz, M. C. A.; Moreira, W. Avaliação das características do TemaTres e Multites para o controle de autoridades nas bibliotecas universitárias. // Scire. 23:2 (jul.-dic. 2017) 63-73. ISSN 1135-3716. 
2 do artigo citado, refere-se aos relacionamentos (equivalência, hierárquicas, associativas) nos tesauros segundo marcos teóricos de revisão de literatura.

Os softwares destinados à construção de tesauros precisam oferecer recursos para atender as necessidades da instituição que o adquirir. Em suas características operacionais, é essencial que o software seja flexível e dinâmico, permitindo manutenção em sua estrutura, como por exemplo, a inclusão ou exclusão de termos da estrutura do tesauro. Isso acontece devido aos avanços tecnológicos e a premissa que as ciências não são engessadas, na verdade se modificam a todo o momento gerando novos saberes e descobertas. Moura et al. (2005) ressaltam que o tesauro é uma ferramenta em permanente construção e isso gera a sua longevidade.

É imprescindível que a instituição invista inicialmente na elaboração de um planejamento, pontuando suas características e necessidades para compra de um software ou mesmo desenvolvimento de um próprio (Gomes; Campos, 2004). A partir do conhecimento desses aspectos há maiores condições para melhores investimentos nos softwares. Referente a esses aspectos, Gomes e Campos (2004, p. 2) ressaltam que é alcançada uma maior segurança para a manutenção da estrutura, evitando a criação de termos anômalos e acrescenta-se que "O que se deve almejar são regras genéricas que contemplem os casos particulares. Isto é: regras prescritivas".

Em seus estudos, Milstead (1991, p.174) relaciona os critérios básicos para um software ser utilizado na construção de tesauros:

Operação em uma plataforma de computador disponível; Integração com software de banco de dados existente; Uma interface amigável; Geração automática de recíprocos; Prevenção de relações conflitantes; Validação de termos em relacionamentos; Suporte de todos os relacionamentos padrão ANSI/NISO; Disponibilidade para adicionar campos de texto; Confirmação de alterações; Uma grande variedade de relatórios e exibições.

A norma utilizada pela autora é ANSI/NISO "Guidelines for Thesaurus Structure, Construction and Use" é datada em 1980, já a norma ANSI/NISO Z39.19 de 2005 "Guidelines for the Construction, Format, and Management of Monolingual Controlled Vocabularies" aponta os seguintes requisitos para um software de gerenciamento de vocabulários controlados, ou seja, as linguagens de indexação, tal qual os tesauros:

1. oferecer suporte, no mínimo, as relações básicas: USE/UP, TG/TE e TR; 2. oferecer suporte para o uso de notas de escopo e de notas históricas; 3 . for- necer tanto exibições hierárquicas quanto alfabéticas; 4. ser, preferencialmente, não-proprietário; 5 . não ser limitado a um único usuário; 6 . ser independente de hardware e operar em sistemas abertos ou em ambiente padrão do sistema operacional; 7. requerer pouco ou nenhum treinamento do usuário; 8 . fornecer documentação detalhada ao usuário (American National Standards Institute/ National Information Standards Organization Z39.19, 2005, p. 99).

É interessante observar que a norma Z39.19 (1980) possui precauções ainda necessárias a um software de construção e manutenção de linguagem de indexação, porém em alguns pontos pressupõe-se que os softwares já estão atualizados nesses quesitos, como operar em uma plataforma de computador disponível, integração com software de banco de dados existente, possuir uma variedade de relatórios e exibições ou mesmo uma interface amigável. Estes são elementos importantes, mas na avaliação dos softwares e diante da atualizada norma Z39.19 (2005), é visível a atenção voltada às relações para a estruturação do tesauro e a inserção dos usuários, diferente da norma Z39.19 (1980) que pontuava elementos mais ao modo operacional do software.

Os problemas de compatibilidade no uso de linguagens de indexação, tais como o tesauro, dizem respeito à como o usuário poderá familiarizar-se intuitivamente e sem ajuda. Sobre isso a ISO 25964-1 (2011, p. vi) considera que "[...] no passado os tesauros foram planejados para profissionais de informação treinados em indexação e busca, hoje existe uma demanda para vocabulários que usuários sem treino irão considerar intuitivos e para vocabulários que permitam inferências por máquinas".

A Norma ISO 25964-1 (2011) recomenda o uso de software de gestão de tesauros para sua construção e manutenção a fim de se evitar erros de digitação e outros erros mais comuns. Para isso, indica em seu item 14 diretrizes específicas para gestão de softwares de tesauro com a orientação de que devem ser capazes de gerenciar, importar e exportar dados. Para a escolha do software indica requisitos a serem seguidos, tais como tamanho e limitações de caractere; relações entre termos e entre conceitos; notas aplicáveis a termos ou conceitos; códigos e notação; etiquetas de vértices ou nó entre termos; status de idiomas; importação e exportação de dados; navegação editorial e apoio; salvaguardas editoriais; e ferramentas de organização.

É preciso destacar dois aspectos importantes na construção e manutenção de tesauros que influenciam na escolha do software de gestão de tesauros: o incremento das relações de equivalên- 
cia e associativas e a construção de tesauros navegáveis. No primeiro aspecto, Boccato (2011, p. 20) ressalta que "o incremento das relações de equivalência e associativas no repertório terminológico possibilita maior aproximação entre o tesauro e a linguagem de busca do usuário, condição essa de suma importância para o adequado desempenho do instrumento terminológico em sistemas automatizados de informação". Com relação ao segundo aspecto, Boccato (2011, p. 21) ressalta que tesauros navegáveis permitem "a migração de tesauros tradicionais a formatos inteligíveis por máquinas, que propicia o intercâmbio e a interoperabilidade entre sistemas de organização do conhecimento".

\section{Softwares de construção e manutenção de tesauros: identificação e análise para discussão de resultados}

Os softwares TemaTres e MultiTes foram identificados nos trabalhos de Campos et al (2006), de Santos, et al. (2013) e Martínez González e Alvite Díez (2014) e selecionados para análise e discussão quanto à proposta de avaliação para construção e manutenção de linguagem de indexação com e para uso no controle de registros de autoridades de catálogos online de bibliotecas.

A análise realizada por Campos et al. (2006) comparou seis softwares, entre eles o TemaTres e o MultiTes, quanto a requisitos divididos em oito grupos: a) Características gerais, b) Tratamento de relações, c) Tratamento de dados, d) Interface/manipulação dos dados, e) relatórios, f) Características de implementação, g) Apoio metodológico, e h) Apoio ao uso. Em Santos et al. (2013) foi realizada a análise dos dois softwares, TemaTres e MultiTes, no desenvolvimento experimental de um micro-tesauro sobre "Cervejas" que avaliou o desempenho metodológico na construção, uso e conversão em vocabulário estruturado para a web semântica. O estudo de avaliação de Martínez González e Alvite Díez (2014) aplicou em seis softwares sete grupos de critérios de avaliação: a) Objetivo do aplicativo, b) Requisitos do sistema, c) Funcionalidades, d) estruturas conceituais e terminológicas compatíveis, e) Gestão da Integridade, f) interoperabilidade da informação, g) Interoperabilidade e integrabilidade do Software, utilizando para os testes de avaliação os tesauros multilíngues: EuroVoc Tesauro multilíngue da União Europeia, UKAT UK Archival Thesaurus, NAL - National Agricultural Library thesaurus - e um microtesauro elaborado ad hoc a partir de EuroVoc.

Os dois softwares serão descritos quanto aos seus requisitos técnicos e funcionalidades opera- cionais e sua aplicabilidade será analisada a partir dos trabalhos de Campos et al (2006), Santos et al. (2013) e Martínez González e Alvite Díez (2014) acompanhados de literatura adicional.

\subsection{TemaTres}

O TemaTres é uma aplicação web utilizada para a gestão de representações linguísticas formais do conhecimento, incluindo vocabulários controlados, taxonomias, tesauros e listas de cabeçaIhos de assunto (Gonzales Aguilar, Ramírez Posada, Ferreyra, 2012; Ferreyra, 2016).

É um software livre (7) desenvolvido por Diego Ferreyra, na Argentina em 2004 (Rodríguez Gairín; Russo Gallo; Sulé Duesa, 2008), e seu sistema também permite desenvolver estruturas de navegação web e funcionar como complemento de um sistema de gerenciamento de bibliotecas, para a gestão da linguagem.

O software TemaTres está disponível para aquisição gratuitamente em sua página web (8) onde também é possível encontrar um tesauro de demonstração construído com o software. A interface de uso do TemaTres é multilíngue e está atualmente em sua versão 2.0 , disponibilizada em espanhol, alemão, francês, inglês, italiano e português.

De acordo com seu manual de utilização (Tematres, 2015), para instalação, o software requer um servidor web que suporte PHP; uma instalação de PHP em funcionamento com versão 4.3 .0 ou superior; e um servidor de bases de dados.

A instalação do software em si, se dá em três passos (Tematres, 2015): 1) realizar o download do arquivo e descomprimi-lo; 2) configurar a conexão com a base de dados MySql no arquivo /include/db.tematres.php; e 3) seguir as instruções de instalação do arquivo.

O software TemaTres permite a criação de linguagens dotadas de controle de vocabulário, entre suas funções permite a criação de relações entre termos, em número ilimitado por termo: relações de equivalência (USE - UP), relações hierárquicas (TG - TE), relações associativas (TR), e permite criar novos tipos de relacionamento entre termos

Possui ferramenta de consistência que permite estabelecer termos equivalentes, não equivalentes e parcialmente equivalentes; atribuição de múltiplas notas aos termos (Notas de aplicação, escopo, explicativa, histórica, bibliográfica etc.); apresenta fluxos de situação termos (termo candidato, termo aceito, termo rejeitado) e possui suporte para vocabulários poli-hierárquicos. Per- 
mite também a navegação entre termos, com sugestão de termos de busca; navegação sistemática e hierárquica (Tematres, 2017).

É possível a exportação completa do vocabulário em formatos que visam à interoperabilidade, tais como, em RDF SKOS-Core, Dublin Core (ISO 15836-2003), BS 8723 (Structured Vocabularies for Information Retrieval), entre outros formatos, para TopicMaps (ISO/IEC 13250:2003), em XML Zthes, MADS (Metadata Authority Description Schema), em formato .txt (ordem alfabética e hierárquica) e SiteMap 0.8 (Tematres, 2017).

O software permite interligar dados entre vocabulários (gerenciar relações com termos de outros vocabulários); auditoria de termos (busca por termos livres e repetidos); administração de usuários e editores e oferece relatórios estatísticos: média de palavras por termo, termos por número de termos genéricos, termos por número de termos específicos, termos por nível de profundidade (Tematres, 2017).

A aplicação web do TemaTres permite visualizar as relações determinadas entre os termos em uma mesma tela.

É importante ressaltar que o software TemaTres possui ferramenta para definição de categorias, com o uso do recurso de Meta-Termos, porém não é possível estabelecer facetas e não permite a indicação de categorias ou macroestruturas, como observado por Santos et al. (2013) que na experiência de aplicação experimental necessitou desenvolver o microtesauro sobre "Cervejas" no MultiTes porque a instalação do TemaTres era mais complexa. Nesse sentido o trabalho aponta que o TemaTres em versão mais atualizada foi escolhido "por conter recursos para geração de listas em formatos como SKOS, XML/BS8723 e XML/DC.

Também, como relatado por Pérez-León e Martínez-González (2010), embora o TemaTres ofereça diversas opções para exportação do tesauro, não há opção para importar tesauros prontos para serem editados no software.

$\mathrm{Na}$ análise realizada por Campos et al. (2006) com o TemaTres na versão de 2005, ressaltou que era o software livre mais completo dentre os demais dessa categoria e que, de maneira geral atendeu de maneira satisfatória a maioria dos requisitos. A análise mais específica de cada requisito apontou algumas limitações tais como: não possui capacidade para referenciar a fonte do termo; não permite a criação de relações definidas pelo usuário que, na análise dos autores, pode ser um problema para a caracterização de diferentes tipos de relações partitivas; não permite a importação nem controle de atualização dos dados; tem problemas na reorganização de hierarquias; permite apenas a emissão de relatório alfabético.

Martínez González e Alvite Díez (2014) descrevem o software TemaTres como uma ferramenta dirigida a desenvolvedores tradicionais de tesauros que torna possível a edição de vocabulários controlados por meio de uma interface web simples e que dispõe de versão web livre para navegar e fazer buscas no vocabulário, além de criar e editar tesauro próprio por meio de mecanismos do software que deverá ser instalado em servidor web local. A avaliação de funcionalidades tais como criação e edição, busca e recuperação, navegação, fusão de tesauros, importação e exportação e colaboração de usuários demonstraram que somente a fusão de tesauros não é possível e que só permite a busca de descritores ou termos preferidos. Por outro lado, dentre os seis tesauros é o único que permite a edição colaborativa de tesauros. Em estrutura conceitual e terminológica apresenta estrutura clássica de tesauro. No que se refere à Integrabilidade destacam como vantagem a simplicidade do software e o enfoque de serviço web que motivam o usuário.

Considerando-se as limitações apontadas pelos autores, observa-se pelas informações atualizadas de Martínez González e Alvite Díez (2014) e do TemaTres em 2017 uma evolução técnica e operacional no TemaTres de instalação e uso, porém, as normas ISO 25693 (2011) e ANSI/NISO Z39.19 (2005) não são citadas. Apesar disso, citam a BS 8723 que fundamentou avanços significativos atualmente existentes nessas duas normas mais atualizadas.

\subsection{MultiTes}

MultiTes Pro é um software proprietário (9), desenvolvido em 1983 para gestão de tesauros, taxonomias e outros vocabulários controlados. A aplicação possui atualmente, uma versão online acessível por um navegador web, além da versão convencional, que deve ser instalada em um computador local. Possui também três serviços complementares que permitem a publicação na web do tesauro, a criação de interfaces sob demanda para pesquisas no tesauro e um conjunto de ferramentas para a publicação do tesauro no servidor da organização para manutenção local. A aplicação está disponível para uso em ambientes Windows 7 ou superior (Multites, 2017) mas também pode ser usado em ambientes Linux (Mandal, 2015).

Entre suas funções, o MultiTes permite a criação de relacionamentos padronizados em conformidade com a norma norte-americana ANSI/NISO 
Z39.19, (USE, UP, BT, NT, RT) com geração automática de relações recíprocas, bem como a criação de notas de escopo e de relações entre termos definidas pelo usuário (Multites, 2017). De acordo com Mandal (2015), MultiTes também realiza validação automática de relações conflituosas entre termos.

O software aplica automaticamente aos top terms e termos não-preferidos formatação padrão (negrito e itálico, respectivamente) para melhor identificação. Permite a criação de poli-hierarquias e tesauros multilíngues. Permite a exportação do tesauro em formatos tais como texto simples, RDF/SKOS, XML, RTF, CSV, HTML e em formato configurável pelo usuário.

$\mathrm{Na}$ análise realizada por Campos et al. (2006) o software MultiTes de 2005 é um software proprietário que atende de maneira mais geral a todos os requisitos analisados e de forma específica observou algumas limitações tais como: o recurso de interface web não é atendido e dificulta a disponibilização em acesso remoto; não permite a geração de um relatório sistemático único com hierarquia de gênero-espécie e parte-todo.

A aplicabilidade experimental do microtesauro sobre "Cerveja" destacou que foi possível criá-lo no MultiTes e convertê-lo para o TemaTres, porém, no processo de migração de dados observou que o arquivo de exportação do Multites não traz elementos suficientes para o reconhecimento do TemaTres e, por isso, as relações entre associativas e de equivalência e notas de escopo precisaram ser manualmente indicadas no arquivo antes da importação.

Martínez González e Alvite Díez (2014) descrevem o software MultiTes como ferramenta comercial disponível pela web e um editor de tesauros com objetivo de construção e manutenção. A avaliação de funcionalidades, acima citadas para o TemaTres, demonstraram que somente a funcionalidade de fusão de tesauros não é possível. Nos critérios de: estrutura conceitual e terminológica apresenta estrutura clássica de tesauro, gestão da integridade, superou todos os testes realizados e de integrabilidade oferece serviços web.

Martínez González e Alvite Díez (2014) concluem que os serviços web com livre acesso oferecidos pelo software TemaTres e outros softwares são úteis para consulta, mas as funcionalidades analisadas não resolvem os problemas de criação e edição de tesauros. Recomendam que seja elaborado um padrão geral de API (10) para a gestão de tesauros.

\section{Considerações finais}

Considerando a importância do controle de vocabulário para as atividades de organização e representação do conhecimento, é certo que a qualidade de seus instrumentos deve ser sempre perseguida. A construção e manutenção de linguagens de indexação tais como o tesauro com uso de softwares adequados é essencial para a garantia de sua permanente atualização e consistência.

Nesse sentido, realizou-se análise dos softwares de construção e manutenção de tesauros TemaTres e MultiTes, que foram citados em avaliações anteriores realizadas por Campos et al. (2006), Santos et al. (2013) e Martínez González e Alvite Díez (2014).

Diante das características identificadas nos dois softwares abordados, tanto por suas informações oficiais quanto na literatura analisada sobre avaliação de softwares de gestão de tesauros, compreende-se que ambos têm as características desejadas para a função a qual se propõem. Porém, em razão de termos deparado com informações conflituosas sobre estas funções, bem como, em razão de que tais softwares disponibilizam atualizações em suas plataformas, podendo no momento presente mostrarem-se com funções mais desenvolvidas ou alteradas, consideramos necessária a realização de aplicação experimental destes softwares para realizar análises mais aprofundadas.

A análise referencial demonstrou que é possível identificar se os softwares possuem os requisitos estabelecidos na norma ANSI/NISO z39.19 (2005) quanto aos critérios de escolha de softwares deste tipo, porém para os requisitos do item 14 da norma ISO 25693 (2011) esta análise não pode ser feita, visto que requer a instalação e teste dos softwares.

Também se coloca como relevante a aplicação experimental para identificar se é possível a realização de edição de tesauros mediante importação de arquivos de autoridades, contendo registros de autoridades em formatos utilizados em sistemas de gestão de catálogos de bibliotecas, posto que tal informação não ficou clara na análise realizada e é de essencial importância para o uso em sistemas de bibliotecas.

Ainda em relação às características desejáveis de um software destinado à construção e manutenção de tesauros, destaca-se de modo enfático a necessidade de investigar, como se pretende fazer em trabalhos posteriores, as funcionalidades relativas à interoperabilidade, como, e.g., a possibilidade de exportação do tesauro em formato SKOS, XML, RDF, entre outros. A adoção

Fujita, M. S. L.; Santos, L. B. P. Dos; Cruz, M. C. A.; Moreira, W. Avaliação das características do TemaTres e Multites para o controle de autoridades nas bibliotecas universitárias. // Scire. 23:2 (jul.-dic. 2017) 63-73. ISSN 1135-3716. 
de um software que não contemple a inserção do tesauro em ambientes de web semântica, além de anacrônica contribui para ações de isolamento entre as diversas bases de dados e unidades de informação. O resultado dessa postura já é conhecido mesmo fora do ambiente web: inoperância do tesauro em função de aspectos que restringem sua aplicação a contextos geográficos e linguísticos mais delimitados.

Quanto ao uso destes softwares em sistemas de bibliotecas, identificou-se que atualmente o TemaTres está sendo utilizado para gestão do Vocabulário Controlado da USP e consta em sua interface de busca por assunto (11). Já quanto ao MultiTes não foi possível identificar experiência de uso em ambientes semelhantes.

\section{Reconocimientos}

Investigação desenvolvida com apoio da FAPESP e CNPq

\section{Notas}

(1) www.taxobank.org

(2) https://bartoc.org/

(3) "Z39.50 é um protocolo de comunicação entre computadores desenhado para permitir pesquisa e recuperação de informação - documentos com textos completos, dados bibliográficos, imagens, multimeios - em redes de computadores distribuídos" (Rosseto, 1997, p.2).

(4) ISO 2709-1981, Documentation - Format for bibliographic information interchange on magnetic tape, criada para o intercâmbio de dados em fitas magnéticas na década de 1960, ainda é utilizada (Assumpção e Santos, 2015).

(5) http://www.niso.org/schemas/iso25964/

(6) http://www.ibict.br/pesquisa-desenvolvimento-tecnologico-e-inovacao/software-para-gerenciamento-de-tesauros-tecer/historico

(7) Software livre é uma denominação que indica que depois de adquirido o software pode ser usado, copiado, estudado, modificado e redistribuído livremente.

(8) http://www.r020.com.ar/tematres/wiki/doku.php?id=tematres:install

(9) Software proprietário é uma denominação que indica que a aplicação está sob proteção legal de direitos autorais, de modo que não é permitido ao usuário copiar, ter acesso ao código-fonte ou redistribuir a aplicação.

(10) Application program interface (API) is a set of routines, protocols, and tools for building software applications. An API specifies how software components should interact. Additionally, APIs are used when programming graphical user interface (GUI) components. Fonte: http://www.webopedia.com/TERM/A/API.html

(11) Cf. http://vocab.sibi.usp.br/pt-br/index.php

\section{Referencias}

Alvite Diez, M. L., Martínez González, M. M. (2015). Armonización de ISO 25964 y SKOS em software de gestión de tesauros: propuesta de análisis. // XII Congreso ISKO España y II Congreso ISKO España-Portugal, 19-20 de no- vembro, 2015, Organización del conocimiento para sistemas de información abiertos. Murcia: Universidad de Murcia, 2015. 266-278. http://www.iskoiberico.org/wp-content/uploads/2015/11/62_Alvite.pdf (2017-03-25).

Assumpção, F. S.; Santos, P. L. V. A. da C. (2015). Representação no domínio bibliográfico: um olhar sobre os Formatos MARC 21. // Perspectivas em Ciência da Informação, 20:1 (2015) 54-74. http://portaldeperiodicos.eci.ufmg.br/index.php/pci/article/view/2054 (2017-0329).

American National Standards Institute/ National Information Standards Organization Z39.19-2005. (2005) Guidelines for the construction, format, and management of monolingual controlled vocabularies. Bethesda, Maryland: NISO Press, 2005. http://www.niso.org/standards/resources/Z39-19-2005.pdf (2017-03-25).

Baritè, M. G. (2011) Sistemas de Organización del Conocimiento: uma tipologia actualizada. Informação \& Informação. 16:3 (2011) 122-139.

Boccato, V. R. C. (2011) Os sistemas de organização do conhecimento nas perspectivas atuais das normas de construção. InCID: R. Ci. Inf. e Doc., 2(1),165-192, jan./jun. http://www.revistas.usp.br/incid/article/view/42340/4601 (2017-03-25).

Broughton, V. (2006) Essential thesaurus construction. London: Facet, 2006.

Campos, M. L. A.; Gomes, H. E. (2006). Metodologia de elaboração de tesauro conceitual: a categorização como princípio norteador. // Perspectivas em Ciência da Informação. 11:3, 348-358. http://portaldeperiodicos.eci. ufmg.br/index.php/pci/article/view/273 (2017-03-25).

Campos, M. L. de A. et al. (2006) Estudo comparativo de softwares de construção de tesauros. Perspectivas em Ciência da Informação. 11:1 (2006) 68-87. http://www. scielo.br/scielo.php?script=sci_abstract\&pid=S141399362006000100006\&lng=p\&tlng=pt (2017-03-25).

Cervantes, B. M. N. (2004). Contribuição para a terminologia do processo de inteligência competitiva: estudo teórico e metodológico. Universidade Estadual Paulista, Marília, 2004. 183 f. Dissertação de Mestrado em Ciência da Informação.

Cervantes, B. M. N.; Fujita, M. S. L. (2012). Uma perspectiva diacrônica de parâmetros e modelos de construção de tesauros. // Cervantes, B. M. N. (Org.). Horizontes da organização da informação e do conhecimento. Londrina: EDUEL, 2012. v. 1, p. 1-18.

Cintra, A. M. M.; et al. (2002) Para entender as linguagens documentárias. 2.ed.rev.ampl. São Paulo: Polis/APB, 2002. $96 \mathrm{p}$.

Dahlberg, I. (1978) Teoria do conceito. // Ciência da Informação. 7:2 (1978) 101-07.

Dextre Clarke, S. G.,; Zeng, M. L. (2012). From ISO 2788 to ISO 25964: the Evolution of thesaurus standard towards interoperability and data modeling. // Information Standards Quarterly. 24:1 (Winter 2012), 20-26. http://www.niso.org/publications/isq/2012/v24no1/clarke/ (2017-03-25).

Gomes, H. E.; Campos, M. L. A. (2004) Tesauro e normalização terminológica: o termo como base para intercâmbio de informações. // DataGramaZero, 5:6 (2004). http://basessibi.c3sl.ufpr.br/brapci/v/a/2052 (2017-0325).

Gonzales Aguilar, Audilio; Ramírez Posada, María; Ferreyra, Diego (2012). Tematres: software para gestionar tesauros. // El profesional de la información. 21:3 (Mayo-Junio) 319-325.

International Organization for Standardization (2011). ISO 25964-1:2011 Information and documentation - Thesauri 
and interoperability with other vocabularies - Part 1: Thesauri for information retrieval. Geneva: International Organization for Standardization, 2011.

International Organization for Standardization (2013). ISO 25964-1:2011 Information and documentation - Thesauri and interoperability with other vocabularies - Part 2: Interoperability with other vocabularies. Geneva: International Organization for Standardization, 2013.

International Organization for Standardization 2788:1986 (1986) Documentation - guidelines for the establishment and development of monolingual thesauri. Geneva: ISO.

Jewett, C. (1853). Smithsonian Catalogue System. Washington, D.C.: Smithsonian Institution. // Mason, Moya K. (2017) Historical Development of Ideas Concerning Library Catalogues: Their Purpose and Organization. http://www.moyak.com/papers/history-library-catalogues.html

Lancaster, F. W. (1995) El control del vocabulario en la recuperación de información. Tradução de Alejandro de la Cueva Martín. València: Universitat de València, 1995. (Educació. Materials, 12).

Mandal, S. (2015). MultiTes: a knowledge organization thesaurus construction tool for college libraries under the University of Burdwan. // International Research Journal of Interdisciplinary \& Multidisciplinary Studies (IRJIMS). 1:8 (September 2015), 63-79. http://irjims. com/files/Sukumar-Mandal.pdf (2017-03-24).

Martínez González, M. M.; Alvite Díez, M. L. Propuesta metodológica de evaluación de gestores de tesauros compatibles con la web semántica. // Anales de Documentación, 17:1. http://dx.doi.org/10.6018/analesdoc.17.1.186271 (2017-03-24).

Martins, M. C. B. (2013) Indexação e controlo da terminologia em bibliotecas do ensino superior politécnico em Portugal: o sistema no Instituto Politécnico de Portalegre. 391p. Departamento de Biblioteconomia y Documentación. Universidad de Salamanca. Salamanca. Tese de Doutorado. http://comum.rcaap.pt/handle/10400.26/7171 (2017-03-25)

Mendes, P. R.; Reis, R. M. dos; Maculan, B. C. M. dos S. (2015). Tesauros no acesso à informação: uma retrospecção. // Revista ACB: Biblioteconomia em Santa Catarina, 20(1), 49-66. http://revista.acbsc.org.br/racb/article/view/980/pdf_110 (2017-03-25).
Milstead, Jessica L. (1991) Specifications for thesaurus software. // Information Processing \& Management, 27:Z/3 (1991), 165-175.

Moura, M. A.; et al. (2005) Linguagens de indexação em contextos cinematográficos: a experiência de elaboração do tesauro eletrônico do cinema brasileiro. // Perspectivas em Ciência da Informação. 10:1, 54-69. http://www.eci.ufmg.br/pcionline/ (2008-04-08).

MULTITES. (2017). MultiTes Pro. // MultiTes: Thesaurus and Taxonomy Authoring Tools. http://www.multites.com/productsPRO.htm (2017-03-25).

Pérez-León, B., \& Martínez-González, M. M. (2010). A comparative study of thesauri tools: a perspective from integrability in information systems. // Filipe, Joaquim y Cordeiro, José (eds.). Proceedings of the 6th International Conference on Web Information Systems and Technologies, WEBIST 2010. Valencia: INS-TICC, 2010. 203-206. https://goo.gl/bTYD7q (2017-03-26).

Rodríguez Gairín, Josep Manuel; Russo Gallo, Patricia; Sulé Duesa, Andreu (2008). A virtual exhibition of open source software for libraries. // 16th BOBCATSSS Symposium 2008, Zadar (Croatia), 28-30 January 2008 http://hdl.handle.net/10760/11151 (2015-12-13).

Rosetto, M. (1997). Uso do protocolo z39. 50 para recuperação de informação em redes eletrônicas. // Ciência da Informação, 26:2 (1997).

Santos, C. A. C. M. dos et al. (2013) Elaboração de vocabulário controlado em formato SKOS usando Tematres: implicações metodológicas e web-semântica. // Dodebei, V.; Guimarães, J. A. C. (Org.). Complexidade e organização do conhecimento: desafios do nosso século. Rio de Janeiro, Marília: ISKO-Brasil, 2013. FUNDEPE. v. 2, p. 205210. http://isko-brasil.org.br/?page_id=42 (2017-03-04).

Scornik, C.; Sosa, O. (2007) Análisis de software para la gestión de lenguajes documentales: caso de estudio: software TemaTres. Corrientes: Universidad Nacional del Nordeste, 2007. http://www.unne.edu.ar/unnevieja/investigacion/com2008/E-020.pdf (2017-03-25)

TemaTres. (2017). TemaTres: gestión de vocabularios controlados. http://r020.com.ar/tematres/wiki/doku.php?id =inicio (2017-03-24).

Enviado: 2017-03-31. Segunda versión: 2017-09-08. Aceptado: 2017-09-15.

Fujita, M. S. L.; Santos, L. B. P. Dos; Cruz, M. C. A.; Moreira, W. Avaliação das características do TemaTres e Multites para o controle de autoridades nas bibliotecas universitárias. // Scire. 23:2 (jul.-dic. 2017) 63-73. ISSN 1135-3716. 
